Provided for non-commercial research and education use. Not for reproduction, distribution or commercial use.

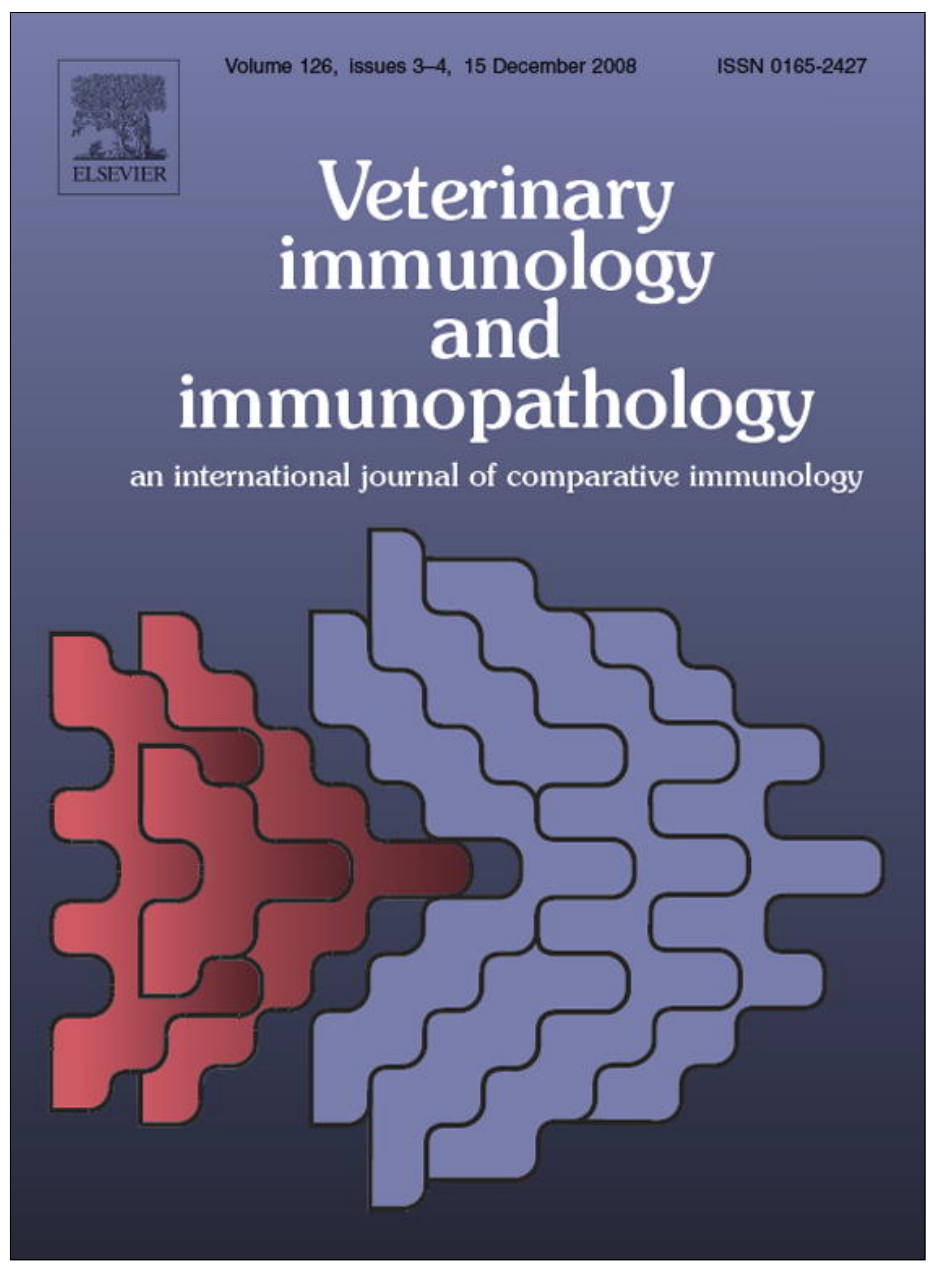

This article appeared in a journal published by Elsevier. The attached copy is furnished to the author for internal non-commercial research and education use, including for instruction at the authors institution and sharing with colleagues.

Other uses, including reproduction and distribution, or selling or licensing copies, or posting to personal, institutional or third party websites are prohibited.

In most cases authors are permitted to post their version of the article (e.g. in Word or Tex form) to their personal website or institutional repository. Authors requiring further information regarding Elsevier's archiving and manuscript policies are encouraged to visit:

http://www.elsevier.com/copyright 


\title{
Langerhans cells in porcine skin
}

\author{
Charles K. Nfon ${ }^{\text {a }}$, Harry Dawson ${ }^{\mathrm{b}}$, Felix N. Toka ${ }^{\mathrm{a}, \mathrm{c}}$, William T. Golde ${ }^{\mathrm{a}, *}$ \\ a Plum Island Animal Disease Center, ARS, USDA, Greenport, NY 11944, United States \\ ${ }^{\mathrm{b}}$ Beltsville Area Research Center, ARS, USDA, Beltsville, MD 20705, United States \\ ${ }^{\mathrm{c}}$ Department of Preclinical Science, Faculty of Veterinary Medicine, Warsaw University of Life Science, Warsaw, Poland
}

\section{A R T I C L E I N F O}

\section{Article history:}

Received 2 April 2008

Received in revised form 27 June 2008

Accepted 16 July 2008

\section{Keywords:}

Porcine

Langerhans cells

Langerin (CD207)

\begin{abstract}
A B S T R A C T
Langerhans cells (LCs) are resident dendritic cells (DCs) of skin and mucosal epithelium. The standard for identifying skin DCs as LCs is expression of langerin (CD207), a surface protein that mediates Birbeck granule (BG) formation upon internalization. Reports of BGs in porcine skin DC are contradictory, due to lack of langerin detection. Here, we present the sequence of porcine langerin/CD207, showing that the predicted porcine protein shares 75\%/86\% amino acid identity/similarity with human. Langerin mRNA was detected in porcine skin DCs by PCR and langerin protein was detected in both isolated skin DCs and skin sections by immunostaining. Approximately, 50-70\% of skin DCs expressed langerin, demonstrating that the majority of porcine skin DCs are LCs. The full length sequence combined with the identification of antibodies reactive with porcine langerin, facilitates the study of LCs in swine, and advances the use of swine for studying skin diseases and infectious disease processes involving skin.
\end{abstract}

Published by Elsevier B.V.

\section{Introduction}

Dendritic cells (DCs) are critical in both early innate and long-term adaptive immune responses (Banchereau and Steinman, 1998). Langerhans cells (LCs) are resident DCs of the epithelium, particularly the epidermis, characterized by the expression of the C-type lectin, langerin (CD207). Langerin is expressed on the cell surface and is internalized to form intracellular, racket-shaped Birbeck granules (BGs) in the cytoplasm (Valladeau et al., 2000), including the dendrites. After encountering antigen, LCs can migrate to regional lymph nodes where, like other DC subsets, they have the unique antigen presenting cell (APC) capacity to induce primary $\mathrm{T}$-cell responses, thereby initiating an adaptive immune response. In mice, it is notable that

\footnotetext{
* Corresponding author at: PIADC, ARS, USDA, P.O. Box 848, Greenport, NY 11944-0848, United States. Tel.: +1 631323 3249;

fax: +1631323 3006 .

E-mail address: william.golde@ars.usda.gov (W.T. Golde).

Abbreviations: DC, Dendritic cell; LC, Langerhans cell; BG, Birbeck granule; CD207, Langerin; APC, antigen presenting cell.
}

langerin is also expressed by non-cutaneous, non-Langerhans cell derived dendritic cell subsets in lymphoid tissue and lung (Douillard et al., 2005; Sung et al., 2006).

The phenotype of human and mouse LCs has been extensively documented (Romani et al., 2006). LCs have also been described in domestic animals including pigs, cattle, sheep, dogs, cats and chickens (Hollis and Lyne, 1972; Khalil et al., 1982; Marchal et al., 1995; Perez-Torres and Ustarroz-Cano, 2001; Romano and Balaguer, 1991). The description of porcine LCs is, however, based on the microscopic identification of BGs in epidermal DCs (Grabbe et al., 1994; Monteiro-Riviere, 1992; Romano and Balaguer, 1991) and LC-like cells derived from monocytes (Paillot et al., 2001). Conversely, we and others did not observe these granules in porcine skin and skin-derived DCs (Bautista et al., 2002; Gregg et al., 1995a). Since langerin is found exclusively in LCs in the skin, antibodies against this molecule have been used to identify human LCs by immunolabelling (Valladeau et al., 2000) and demonstration of expression of langerin has become the standard for designating skin dendritic cells as LCs.

We have previously characterized DCs isolated from porcine skin as coexpressing CD1 and CD172. These cells 
also express high levels of MHC class II, CD80/86 and S100 antigen but lack expression of lymphoid specific proteins such as CD3, CD4, CD8 and CD21 (Bautista et al., 2002). However, in the absence of specific antibodies to porcine langerin, the further characterization of these porcine skin DCs has been limited relative to classifying Langerhans cells.

Pigs have important physiological and immunological similarities with humans. Therefore, they have been proposed as important models in human medicine and potential organ donors for xenotransplantation (Dunning et al., 1994; Sablinski et al., 1997; Sim et al., 1999). Furthermore, pigs are an important food source, necessitating high health standards through control and eradication of infectious diseases. As such, an understanding of the immunological properties of porcine LCs and other porcine APCs will be valuable in both human and veterinary medicine.

The objective of the present work was to identify langerin expressing skin dendritic cells in swine by multiple assays. We have sequenced the full length porcine langerin gene and here we present a comparative analysis of the predicted protein sequence relative to the langerin protein in human and other animal species. The predicted porcine CD207 protein shares high amino acid identity/similarity with human $(75 \% / 86 \%)$ and lesser amino acid identity/similarity to mouse $(63 \% / 77 \%)$ and rat $(63 \% / 76 \%)$ CD207. Further, we show langerin expression in porcine skin DCs by quantitative realtime reverse transcriptase polymerase chain reaction (qrRT-PCR), with mRNA levels more than 100-fold higher in putative LCs than in porcine monocyte-derived DCs (MoDCs). We then tested nine anti-human langerin monoclonal antibodies (MAb) by flow cytometry showing eight reacted with porcine langerin expressed in isolated skin DCs, with six antibodies binding to more than $50 \%$ of porcine skin DCs. In addition, epidermal DCs in skin sections stained with anti-human langerin by immunohistochemistry (IHC) and immunofluorescent microscopy (IF). We have thus identified the tools necessary for further studies of the role of porcine LCs in immune responses and immunopathology of disease.

\section{Materials and methods}

\subsection{Langerin (CD207) cloning, sequencing and structural and functional analysis}

Cloning primers were designed using human (Genbank NM_015717)/bovine (Genbank XM_588243) cross reactive sequence using the Primer Express software package (Applied Biosystems, Foster City, CA) and are listed in Table 1. Duplicate $50 \mu$ l PCR reactions using these primers were performed using a cDNA pool made from eight porcine tissues and $2 \times$ PCR master mix (Abgene/Thermo, Waltham, MA). These products were amplified by 45 cycles of PCR on an iCycler thermocycler (Biorad, Hercules, CA). Primers and products under $100 \mathrm{bp}$ were removed from amplified material with the Qiaquick PCR purification kit (Qiagen, Valencia, CA). The presence of a single band of expected size was determined using the Agilent Bioanalyzer 2100 and a DNA 1000 Labchip kit (Agilent Technologies, Palo Alto, CA). These products were then amplified for sequencing using
Table 1

Primers used in the cloning of porcine CD207

\begin{tabular}{lll}
\hline Sequence & $\begin{array}{l}\text { Position } \\
\text { in cDNA }\end{array}$ & Exon \\
\hline Cloning & & \\
F 5'-GCAGCCAGAAGCACCTGTG-3' & NA & 1 \\
R 5'-TGCCCATAAACCAAGGATAGAGA-3' & $235-213$ & 2 \\
F 5'-CCTGGTCGCCTCCATCCT-3' & $183-200$ & 2 \\
R 5'-GTGCATCAGCTTCCTTCACACT-3' & $433-412$ & 3 \\
F 5'-CCTGAGTTCCGAAGTCAATAGGA-3' & $297-319$ & 2 \\
R 5'-GACGTTCCCCCGGAGTACT-3' & $645-626$ & 4 \\
F 5'-ATGACATTCTCCAGATGATGTCTCAA-3' & $593-618$ & 4 \\
R 5'-GGGACATAGGGTCGTTTACAGATAA-3' & $1000-977$ & 6 \\
F 5'-GGTTCTGGATTCCAGGTGAGC-3' & $863-883$ & 6 \\
R 5'-CTGGAGTATGAAGGAGAGAACACCATT-3' & NA & 6 \\
PCR assay & & \\
F 5'-GGAATGATGCCCCCTGTGA-3' & $944-962$ & 6 \\
R 5'-CGTTGGAACTTAAAGTGCAAGCT-3' & $1030-1052$ & 6 \\
Probe 5'-CGACCCTATGTCCCATCAGAACCGTG-3' & $988-1013$ & 6 \\
\hline
\end{tabular}

Big Dye Terminator v3.1 cycle sequencing kit (Applied Biosystems) on an iCycler thermocycler (Biorad). After purification by Performa spin columns (Edge Biosystems, Gaithersburg, MD), amplicons were spun dry, re-suspended in Hi-Di Formamide (Applied Biosystems), denatured at 95 degrees for 2 min and sequenced on a 3100 Genetic Analyzer 16 capillary array (Applied Biosystems) at the Environmental Microbial Safety Laboratory at the Animal and Natural Resources Institute, USDA, Beltsville, MD.

A consensus sequence for each gene target was assembled from four independent sequence reads, and then compared to the human reference sequences. Contiguous sequences were assembled using the CAP3 sequence assembly program (http://pbil.univ-lyon1.fr/cap3.php). The predicted exon structure was determined by BLAST searching the deduced cDNA assembly against human and bovine genomic DNA (http://www.ncbi.nlm.nih.gov/ blast/). The predicted protein structure was compared to other mammalian CD207 homologues by a translated BLAST search of the non-redundant protein database.

A phylogenetic tree comparing the predicted porcine CD207 protein with putative full-length amino acid sequences of known mammalian CD207 proteins was generated using Clustal X (v2.0.8 European Bioinformatics Institute). The alignment was compared by Bayesian Markov chain Monte Carlo algorithms employing a strict molecular clock model and a chain length of 10,000,000 using BEAST v1.4.7 software in auto optimize mode (Drummond and Rambaut, 2007). Two independent runs of 2500 iterations were generated. The output was used to generate a consensus tree using Genious Pro (v3.7.1 Biomatters Ltd., Auckland, New Zealand). Calculated posterior probability of monophylogeny is displayed on the nodes. Functional domains and glycosylation site predictions were determined using the SMART architecture program (http://smart.embl-heidelberg.de/).

\subsection{Animals, isolation of skin dendritic cells, epidermal keratinocytes and alveolar macrophages}

Yorkshire pigs (female) aged 6-8 months were purchased from Animal Biotech Inc., Danboro, PA and allowed 
1 week to acclimatize and all animals were deamed healthy before the start of experiments. All procedures performed on these animals were approved by the Plum Island Animal Disease Center (PIADC) Institutional Animal Care and Use Committee.

Skin DCs were isolated following a published protocol (Bautista et al., 2002; Gregg et al., 1995b). Briefly, the skin of the entire back of a euthanized pig was washed, depilated with Nair (Carter Wallace Inc., New York, NY), surgically scrubbed, and disinfected. Skin layers (0.1$0.15 \mathrm{~mm}$ thick) were cut with a microtome blade and collected into Basal Eagle Medium (BEM, Invitrogen, Carlsbad, CA,) supplemented with antibiotics (BEM-AB). Skin layers were washed twice in BEM-AB and then cultured (epidermis up) in RPMI-1640 containing $0.1 \mathrm{mM}$ non-essential amino acids, $0.3 \mathrm{mg} / \mathrm{ml}$ L-glutamine, $5.5 \times 10^{-5} \mathrm{M} \beta 2$-mercapthoethanol, $1 \mathrm{mM}$ sodium pyruvate, $4 \mathrm{mM}$ dextrose, and $10 \%$ heat-inactivated fetal bovine serum (culture medium) supplemented with $10 \%$ heatinactivated autologous porcine serum in $150 \mathrm{~cm}^{2}$ cell culture petri dishes at $37{ }^{\circ} \mathrm{C}, 5 \% \mathrm{CO}_{2}$ and $85 \%$ relative humidity overnight. Skin DCs that migrated from skin were recovered as previously described (Bautista et al., 2002).

Epidermal keratinocytes (EKs) were isolated from skin layers remaining after overnight skin DCs migration. The method for EK isolation was modified from a published protocol (Gray and Yardley, 1975). Briefly, small pieces of skin layers were digested in $0.05 \%$ trypsin-EDTA (Invitrogen) at $37{ }^{\circ} \mathrm{C}$ for $90 \mathrm{~min}$ with shaking. The cell suspension was withdrawn and more trypsin-EDTA added to the skin layers at $37^{\circ} \mathrm{C}$. After another $90 \mathrm{~min}$, the reaction was stopped by adding 10\% FBS and the cell suspension filtered to remove skin debris. The isolated cells were suspended in culture medium and viability assessed by trypan blue exclusion. Residual (CD1+) skin DCs in the EKs suspension was determined by flow cytometry.

Porcine alveolar macrophages (PAMs) were collected by lung lavage as previously described (Bautista et al., 2002). Briefly, lungs removed from a euthanized pig were filled with PBS containing antibiotics, gently massaged and the medium collected into a beaker. After centrifugation, contaminating erythrocytes were lysed in hypotonic buffer, followed by washes in PBS and the PAMs resuspended in culture medium.

For PBMC isolation, heparinized blood was diluted in an equal volume of PBS and layered over a separation gradient (Lymphoprep $^{\mathrm{TM}}$, Life Technologies, Grand Is., NY) following the manufacturer's protocol. Negative selection with Dynal beads (Dynal Corp., Invitrogen, Carlsbad, CA) was used following the manufacturer's protocol to deplete
PBMCs of $\mathrm{T}$ and $\mathrm{B}$ cells, and the remaining cells tested for langerin expression. Briefly, PBMCs were incubated with mouse antibodies to porcine CD3 and CD21 to eliminate T and B cells respectively and enrich for blood DCs. Beadspositive cells were then separated with a magnetic field. Isolated cells were used for flow cytometry and RNA isolation for RT-PCR.

\subsection{Polymerase chain reaction}

Cells for RNA extraction ( $5 \times 10^{5}$ skin DCs, PAMs or EKs) were preserved in Trizol (Invitrogen) and stored at $-70{ }^{\circ} \mathrm{C}$. As a negative control for langerin, RNA was also extracted from porcine monocyte-derived DCs differentiated from monocytes as previously described (Bautista et al., 2007). Total cellular RNA was isolated using the Invitrogen protocol for Trizol reagent and cDNA synthesized from approximately $1 \mu \mathrm{g}$ of the extracted RNA as previously reported (Bautista et al., 2005).

For TaqMan realtime PCR, samples were analyzed in duplicates, with approximately $10 \mathrm{ng} /$ well of cDNA used in a PCR mix of $25 \mu \mathrm{l}$ containing optimal concentrations of primers, probes (Table 2) and the TaqMan universal PCR mastermix (Applied Biosystems, Foster City, CA) following the manufacturer's protocol. Data was acquired in an $A B I$ PRISM 7700 Sequence Detection System (Applied Biosystems). The level of langerin mRNA expression for each sample was determined by the comparative cycle threshold (Ct) method (user bulletin 2, Applied Biosystems), using ubiquitin gene as an endogenous control to normalize the data. Data for MoDCs was then used as a calibrator for the langerin expression in skin DC, EK and PAM samples applying the formula $2^{-\Delta \Delta \mathrm{Ct}}$ (user bulletin 2, Applied Biosystems) to calculate the fold difference in mRNA. MoDCs were generated to yield DCs of high purity for preparation of polyA RNA as blood DCs are remarkably rare and purification above $50 \%$ very difficult.

\subsection{Antibodies}

All antibodies raised against human Langerhans cells and reactive with human langerin, clones 808E10.01, 310F7.02, 306G9.01, 817G7, and 122A11 (all mouse IgG1) and antibodies raised against recombinant human langerin and reactive with both mouse and human langerin, clones 929F3.01, 923B7, 926G4, 929F7 (all rat IgG2a) were provided by Dendritics (Lyon, France). Antibodies to porcine CD1 (clone 76-7-4, mouse IgG2a) and CD172 (formerly SWC3a) (clone 74-22-15, mouse IgG2b) were purchased from Southern Biotech (Birmingham, AL).

Table 2

Primers and probes used for real-time PCR

\begin{tabular}{lll}
\hline Primer or probe name & Final concentration $($ nM) \\
\hline p-CD207-44F & Sequence $\left(5^{\prime}-3^{\prime}\right)$ & 900 \\
p-CD207-152R & GGAATGATGCCCCCTGTGA \\
p-CD207-88T & CGTTGGAACTTAAAGTGCAAGCT \\
p-UBC-47F & CGACCCTATGTCCCATCAGAACCGTG \\
p-UBC -126R & GCGCACCCTGTCTGACTACA \\
p-UBC-79T & AGATCTGCATCCCACCTCTGA & 900 \\
\end{tabular}

${ }^{a}$ p, porcine; F, forward primer; R, reverse primer; $T$, TaqMan TET-labeled probe. UBC, ubiquitin (house keeping gene). 


\subsection{Flow cytometry}

The expression of the cell-specific antigens was analyzed by flow cytometry. Briefly, $1 \times 10^{6}$ cells were washed in chilled FACS buffer (PBS with $0.3 \%$ BSA and $0.1 \%$ sodium azide) prior to being stained for $30 \mathrm{~min}$ on ice with optimal dilutions of the specific antibodies $(10 \mu \mathrm{g} / \mathrm{ml}$ for all the anti-langerin antibodies) or their respective isotype controls. For intracellular staining, cells were fixed and permeabilized with BD cytoperm/cytofix (BD Biosciences, San Jose, CA, USA) according to manufacturer's protocol prior to incubation with specific antibodies. For double staining, cells were first stained for CD1, fixed and permeabilized before staining for langerin. Either goat anti-mouse IgG-FITC (STAR117F, Serotec), goat anti-mouse IgG1-PE (STAR81PE, Serotec) or goat anti-rat IgG-PE (AQ200H, Chemicon International, Temecula, CA, USA) were used as secondary antibodies for langerin staining. Double staining of skin DCs was also performed with CD1 and CD172 and the langerin+ population expressed as a percentage of the CD1/CD172 double positive cells. Data was acquired on a FACSCalibur ${ }^{\mathbb{R}}$ flow cytometer using the Cell Quest ${ }^{\circledR}$ software (BD Biosciences) and expressed as percent positive cells for each marker after background correction with isotype controls.

\subsection{Immunohistochemistry}

Cryosections of porcine skin were fixed with BD cytoperm/cytofix at $4{ }^{\circ} \mathrm{C}$ for $30 \mathrm{~min}$, washed with BD permwash (BD Biosciences) and endogenous peroxidase quenched with $3 \%$ hydrogen peroxide for $5 \mathrm{~min}$. The slides were then blocked with blocking buffer ( $2 \%$ porcine serum, bovine serum albumin, fetal bovine serum and nonfat skimmed milk dissolved in BD perm/wash buffer) at room temperature for $60 \mathrm{~min}$. Optimal concentrations of primary antibodies $(25 \mu \mathrm{g} / \mathrm{ml}$ anti-human langerin Mab clone $306 \mathrm{G} 9.01$ and $310 \mathrm{~F} 7.02$ or mouse IgG1 as isotype control) diluted in blocking buffer were then added to individual slides for $24 \mathrm{~h}$ at $4{ }^{\circ} \mathrm{C}$. The slides were further incubated with anti-mouse IgG ImmPress ${ }^{\mathrm{TM}}$ reagent (Vector Laboratories, Burlingame, CA, USA) at room temperature for $30 \mathrm{~min}$. AEC substrate (Vector Laboratories) was added for $5 \mathrm{~min}$, washed and the slides counterstained in Mayer's hematoxylin for $30 \mathrm{~s}$. The slides were then mounted in GVA mount (Zymed, San Francisco, CA, USA) and sealed with a cover slip for viewing on a microscope.

\subsection{Immunofluorescent microscopy}

Cryosections of porcine skin and cytospins of isolated skin DCs were fixed with BD cytoperm/cytofix at $4{ }^{\circ} \mathrm{C}$ for $30 \mathrm{~min}$. The slides were then blocked with blocking buffer ( $10 \%$ porcine serum in BD perm/wash buffer) at $37{ }^{\circ} \mathrm{C}$ for $60 \mathrm{~min}$. Optimal concentrations of primary antibodies (25 $\mu \mathrm{g} / \mathrm{ml}$ anti langerin clone 929F3 together with anti porcine CD1 clone 76-7-4 for double staining or mouse/rat IgG2a as isotype controls) diluted in blocking buffer were then added to individual slides for $24 \mathrm{~h}$ at $4{ }^{\circ} \mathrm{C}$. The slides were further incubated with anti-rat IgG-Alexa Fluor ${ }^{\circledR} 594$ and anti-mouse IgG2a-Alex Fluor ${ }^{\circledR} 488$ (Invitrogen) in blocking buffer at $37^{\circ} \mathrm{C}$ for $60 \mathrm{~min}$ to detect langerin and CD1 respectively or the corresponding isotype controls. Note that the slides were washed between each step with BD perm/wash (BD Biosciences). Some slides were incubated with a nuclear stain (TOPRO-iodide, Invitrogen) for $5 \mathrm{~min}$ to reveal the architecture of the skin section. Slides were then mounted using a mounting medium for fluorescent microscopy (KPL, Gaithesburg, MD, USA), viewed on an OLYMPUS BX40 fluorescent microscope (Scientific Inc., Piscataway, NJ, USA) and images were captured with a DP-70 digital camera and analyzed using DP-BSW v2.2 software (Scientific Inc., Piscataway, NJ, USA).

\subsection{Internalization of anti-langerin antibody}

The internalization assay was performed as previously described (Valladeau et al., 1999). Briefly, skin DCs were washed in PBS containing 1\% fetal calf serum (PBS-F) and stained with anti-langerin clone $122 \mathrm{~A} 11$ on ice for $30 \mathrm{~min}$. After washing twice with PBS-F, cells were further stained with biotin anti-mouse IgG (eBioscience) on ice for $30 \mathrm{~min}$. As time zero, $5 \times 10^{5}$ cells were stained with streptavidinPE (PharMingen) for $30 \mathrm{~min}$. The rest of the cells were either incubated at $37{ }^{\circ} \mathrm{C}$ or on ice for 15 and $30 \mathrm{~min}$. Some cells were fixed and incubated at $37^{\circ} \mathrm{C}$ to assess the offrate of the antibody at this temperature. At indicated timepoints, cells were washed in chilled FACS buffer and stained with streptavidin-PE as above. Finally, cells were analyzed by flow cytometry and antibody internalization measured as percentage decrease in mean fluorescence intensity relative to control samples kept on ice.

\section{Results}

\subsection{CD207 cloning and predicted structural and functional analysis}

The assembly of the porcine langerin gene (CD207) yielded a 1069 bp cDNA sequence containing the entire predicted coding region (Genbank EU442798). Nucleotides 1-24 contains part of the $5^{\prime}$ untranslated region. Nucleotides 25-1011 contain the coding regions. Nucleotides 1012-1069 contain part of the $3^{\prime}$ untranslated region. The predicted exon locations are as follows; exon 1 bp 1-98, exon 2 bp 99-217, exon 3 bp 218-592, exon 4 bp 593-744, exon 5 bp 745-863, and exon 6 bp 864-1096. The open reading frame of porcine $\mathrm{CD} 207$ is predicted to encode a 329 amino acid protein (Fig. 1). The predicted molecular weight is $37.5 \mathrm{kDa}$ and if the protein is fully glycosylated, the molecular weight is predicted to be $40 \mathrm{kDa}$. Porcine CD207 is closest in size to the bovine (329) and human (328) proteins followed by the mouse (331), rat (332) and canine (200) proteins. The predicted canine protein is missing an internal stretch of 129 amino acids spanning the predicted membrane-proximal neck domain. The predicted porcine CD207 protein shares high amino acid identity/similarity with canine CD207 (76\%/83\%), human (75\%, 86\%) and bovine (73\%/84\%) CD207, and lesser amino acid identity/similarity to mouse $(63 \% / 77 \%)$ and rat $(63 \% /$ 76\%) CD207 proteins. 


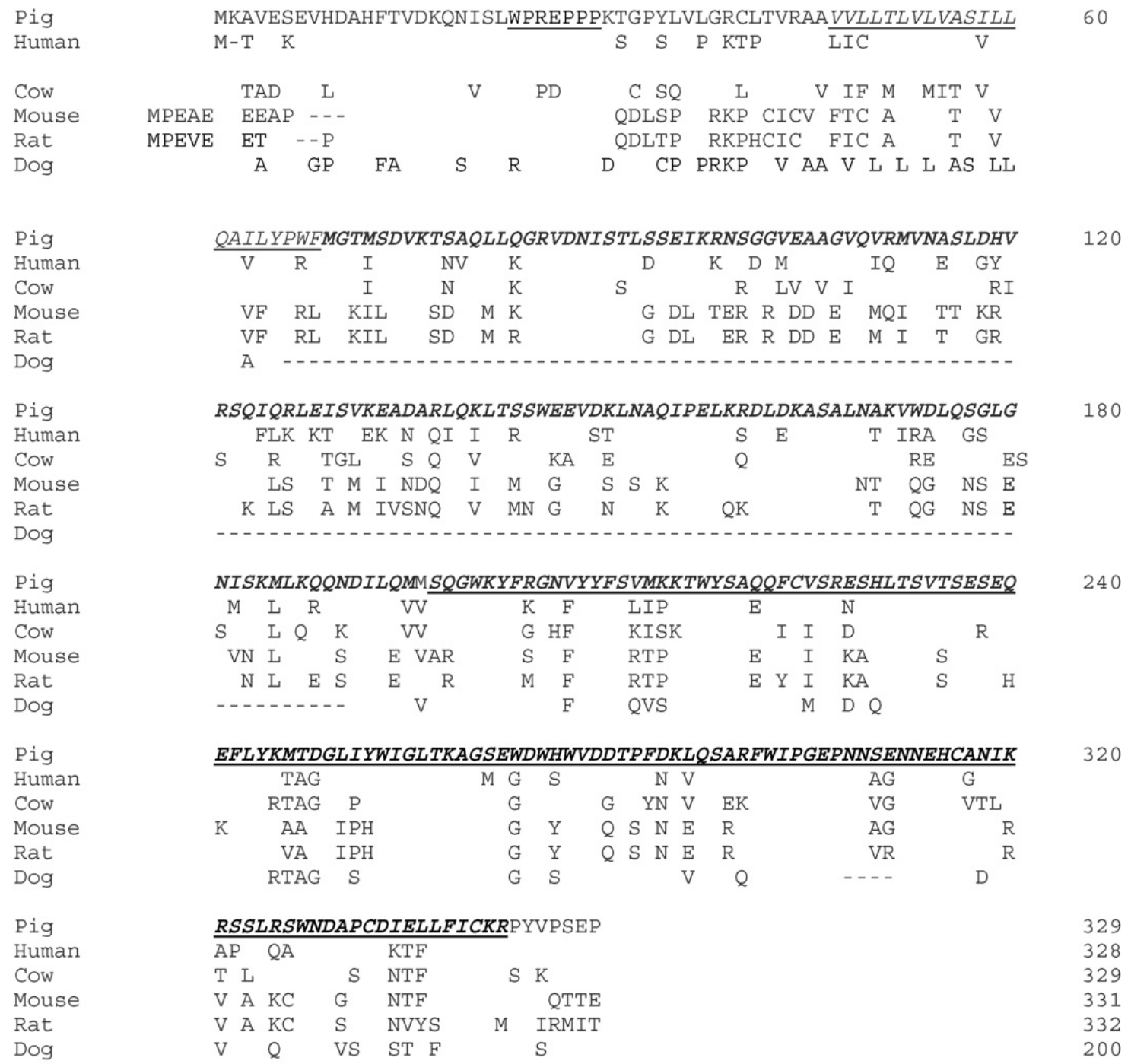

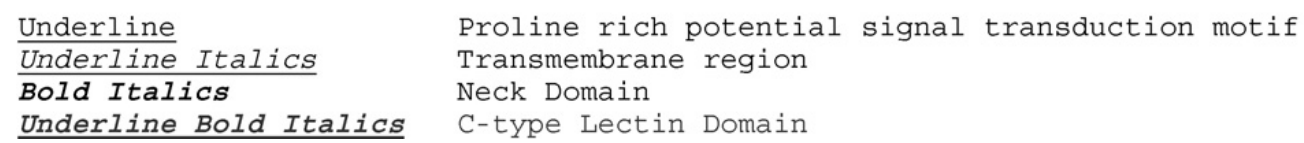

Fig. 1. Multiple sequence alignment of the predicted sequence deduced from porcine CD207 cDNA. Functional domains were predicted as described in the Materials and Methods section. The proline rich potential signal transduction motif is underlined. The transmembrane region is underlined and italicized. The neck domain is boldfaced and italicized. The C-type lectin domain is underlined, italicized and boldfaced. Sequences used in the alignment are: Pig (ACC63373), Human (NP_056532), Cow (XP_588243), Mouse (NP_659192), Rat (XP_578352) and Dog (XP_855502).

Amino acids 23-29 of the predicted porcine CD207 molecule encode a proline rich potential signal transduction motif (WPREPPP) that is conserved across species. In other proteins this motif associates with $\mathrm{SH} 3$ domain proteins and is involved in endocytic vesicle trafficking and cytoskeletal movement. Amino acids 46-68 encode the predicted transmembrane region. Amino acids 69-195 encode the membrane-proximal neck domain. This area displays a moderate level of conservation among species. According to the manufacturer, the rat anti-human monoclonal 929F3 used in this study was generated to an epitope in the neck domain. Two potential $\mathrm{N}$ glycosylation sites occur in the membrane-proximal neck domain at amino acids 114 and 181. Amino acids 196-321 encode the C-type lectin domain. The tryptophan residue essential for human Bierbeck granule formation is conserved at position 263 (Verdijk et al., 2005). Amino acids 286-288 encode a conserved Glu-pro-asn (EPN) motif that is essential for mannose recognition (Stambach and Taylor, 2003). The C-type lectin domain contains conserved glutamic acid (262) and asparagine (289) residues and glutamic acid (286), asparagine $(288,308)$ and aspartic acid (309) residues that form the $2 \mathrm{Ca}^{2+}$ binding sites in the human CD207 protein (Stambach and Taylor, 2003).

The evolutionary relationship between several mammalian homologues/orthologues is shown in Fig. 2. Two independent runs of 2500 iteration were generated and they agreed in probability, ordering of branches and 


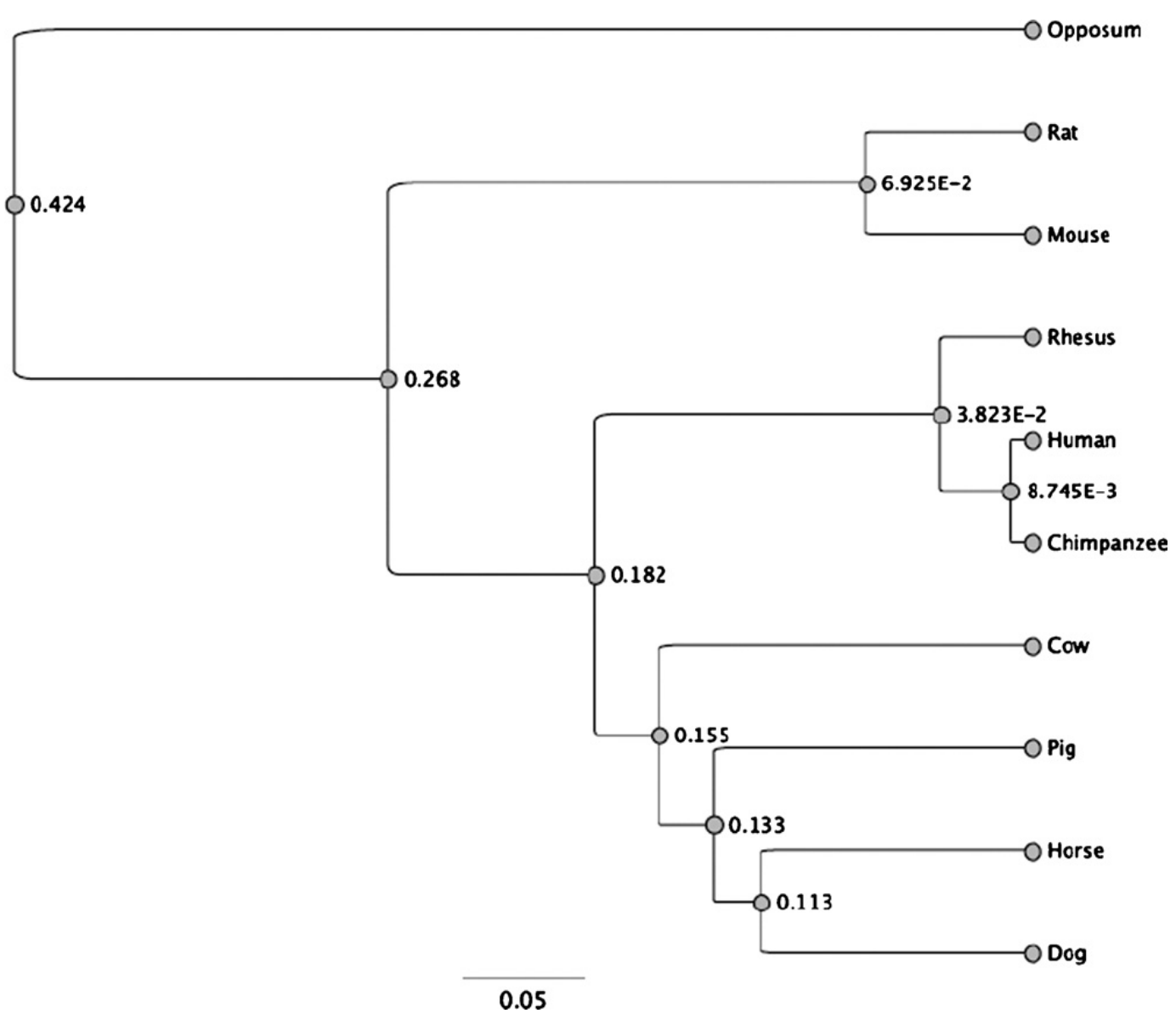

Fig. 2. Phylogenetic tree of mammalian CD207 homologues/orthologues. Bayesian estimation of the evolutionary relationship among predicted sequences were estimated as described in the Materials and Methods section. One of two runs is displayed. Calculated posterior probability of monophylogeny is displayed on the nodes. The bar represents 5\% estimated phylogenetic divergence. Sequences used in the construction are: Opossum (XP_00137661), Dog (XP_855502), Rat (XP_578352), Mouse (NP_659192), Cow (XP_588243), Horse (XP_001492626), Pig (ACC63373), Rhesus Monkey (XP_001100466) Human (NP_056532) and Chimpanzee (XP_001144168).

evolutionary distance. One tree is shown in Fig. 2. Porcine and equine CD207 form a separate clade. Porcine CD207 is predicted to be closer to primate CD207 than rodent CD207, which forms a separate subclade with opossum CD207.

\subsection{Transcription of langerin $m R N A$ in porcine skin DCs}

To demonstrate the transcription of langerin in porcine skin DCs, we designed a qrRT-PCR assay to detect langerin specific mRNA. The level of langerin-specific mRNA in skin DCs was compared to that in PAMs and EKs, using MoDCs as the calibrator. Skin DCs $(n=3)$ expressed 105.3-fold more langerin mRNA than MoDCs. Though analysis of PAMs and EKs from three different animals showed 16.7and 12.5-fold (respectively) more expression of langerin mRNA than MoDCs derived from the same three animals, these levels were significantly lower when compared to skin DCs.

\subsection{Monoclonal antibody reactivity with porcine langerin}

Of the nine monoclonal anti-human langerin antibodies tested for cross-reactivity with porcine langerin, three antibodies representing the difference in reactivity with intracellular verses extracellular langerin are shown in Fig. 3. Monoclonal antibodies 929F3 and 310F7.02 stained intracellular epitopes of langerin while clone 122A11 stained epitopes on the cell surface (Fig. 3). This dichotomy was reported by Saeland and colleagues (Valladeau et al., 1999) for another anti-langerin antibody, DCGM4, in the staining of human LCs. We previously reported DCGM4 does not cross-react with porcine skin DCs (Bautista et al., 2002). Analysis of the cellular localization of the epitopes recognized by the antibodies used here with the human langerin protein is available from the supplier, Dendritics (Lyon, France).

Two antibodies showed little or no reactivity with porcine langerin. Specifically, clone 808E10.01 showed moderate reactivity with a low mean fluorescence intensity (MFI) while clone $817 \mathrm{G} 7$ gave signal close to background level (Fig. 4). Seven clones reacted with 47$60 \%$ of DCs derived from porcine skin and four of these had high MFI (Fig. 4).

Porcine skin DCs are CD1 and CD172 double positive (Bautista et al., 2002). Therefore, to estimate the proportion of skin DCs that express langerin, we analyzed the CD1/CD172 double positive cells with anti-langerin and show that the percentage of skin DCs expressing langerin ranged from $32 \%$ to approximately $80 \%$ (Table 3 ). Indeed, 


\section{Porcine skin DC reactivity with mouse anti-human langerin antibodies \\ Permiablized Cells}

Non-permiablized Cells
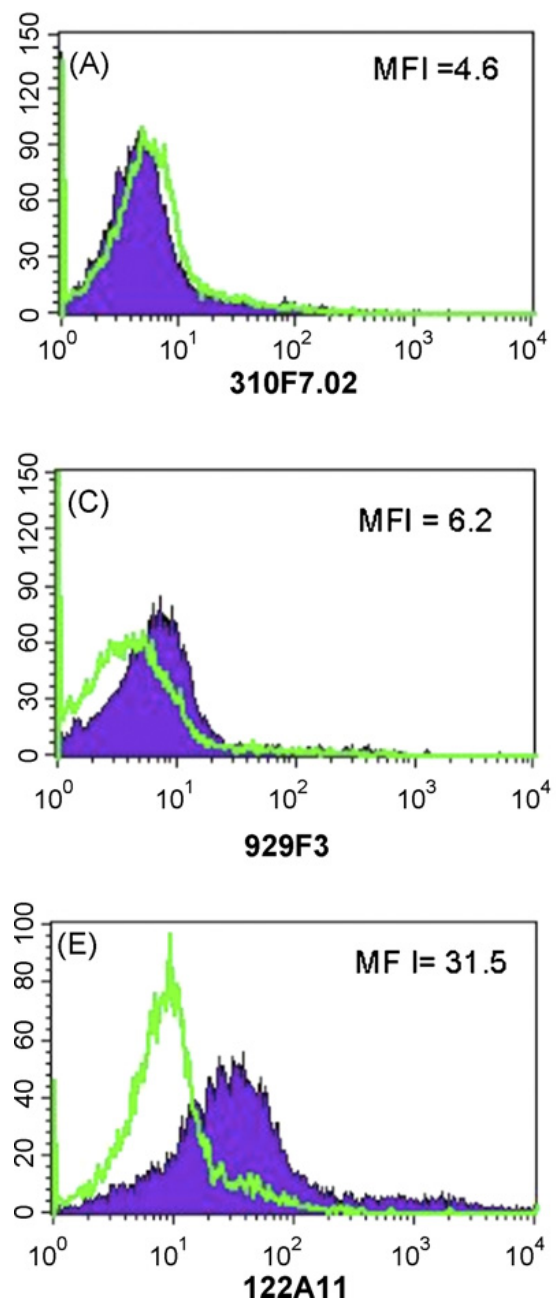
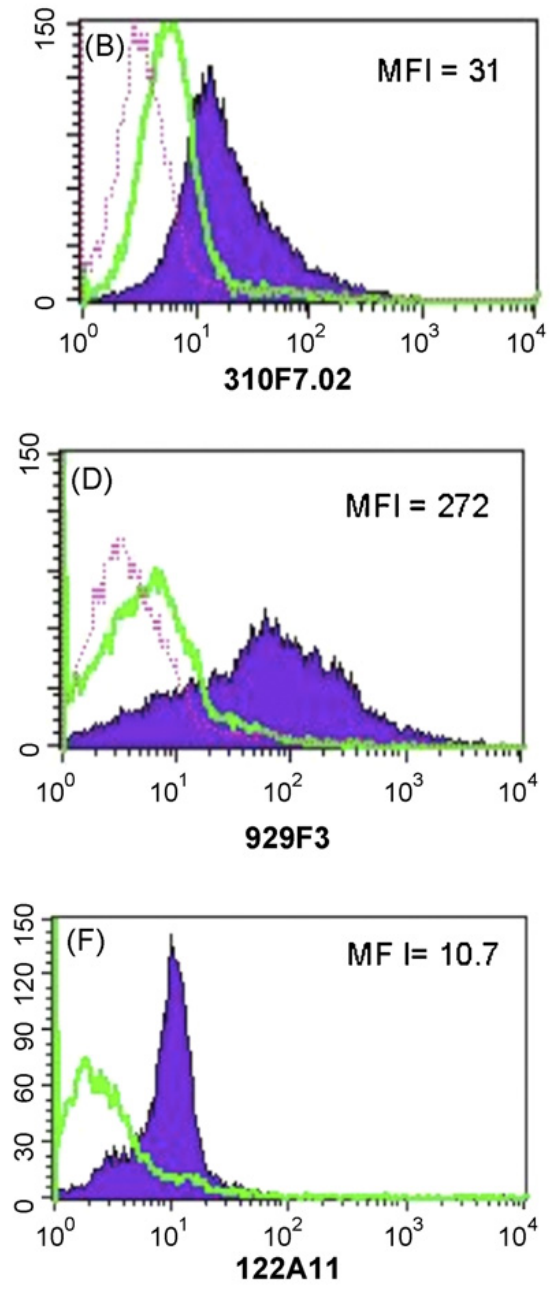

Fig. 3. The fluorescence of porcine skin-derived DCs (skin DCs) stained with representative clones of anti-human langerin antibodies. Cells derived from overnight culture of porcine skin biopsies were stained either permeabilized or non-permeabilized with optimal concentrations of anti-human langerin antibodies and fluorescence analyzed on a FACS Calibur ${ }^{\circledR}$ flow cytometer. The histogram with broken lines represents unstained cells while the solid lines and the filled histograms represent the isotype control and the langerin stained cells respectively for Clone $310 \mathrm{~F} 7.02$ and $929 \mathrm{~F} 3$ specific for an intracellular epitope and clone 122A11, specific for an extracellular epitope of langerin. Geometric mean fluorescence intensity (MFI) is at the right of each plot.

double staining of the skin DCs with anti-CD1 and antilangerin clones 306G9.01 and 929F3.01 revealed reactivity with $50-73 \%$ of the CD1 positive cells in these two examples (Fig. 5A). Notably, these antibodies stained none or a negligible percentage of PAMs (Fig. 4A), blood DCs (Fig. 5B) and MoDCs (data not shown). Blood DCs were about $30 \%$ of the CD1 positive cells (reactive with both anti-CD1 and anti-CD172) after negative selection for CD3 and CD21, yet only $8 \%$ of the CD1 cells were detected with anti-langerin. It is worth noting that though blood DCs form the bulk of CD1/CD172 double positive cells, other mononuclear cells may also show this phenotype. Similarly, less than $8.0 \%$ of EKs isolated from the same skin biopsies as the DCs were stained (Fig. 4A). The small percent of EKs that stained with anti-langerin might represent residual skin DCs, as these were also CD1 positive (Fig. 5B).
3.4. Anti-langerin antibodies stain LCs in porcine skin by immunohistochemistry and immunofluorescent microscopy

Two anti-human langerin Mabs are recommended by the manufacturer for use in IHC, clones 306G9.01 and 310F7.02. We tested these antibodies for reactivity with porcine skin and show both antibodies reacted with LCs in the skin (Fig. 6). However, clone 310F7.02 apparently stained LCs in skin sections more intensely (Fig. 6A and B) compared to clone 306G9.01 (Fig. 6C and D). The langerinpositive cells were concentrated in the epidermis and isotype controls were negative (Fig. 6E).

Clone 929F3 is also recommended by the manufacturer for use in IHC on paraffin sections. We obtained a relatively high fluorescent intensity with this antibody by flow cytometry (Figs. 4B and 5A, right hand panel). We therefore used this antibody to stain isolated skin DCs and frozen 

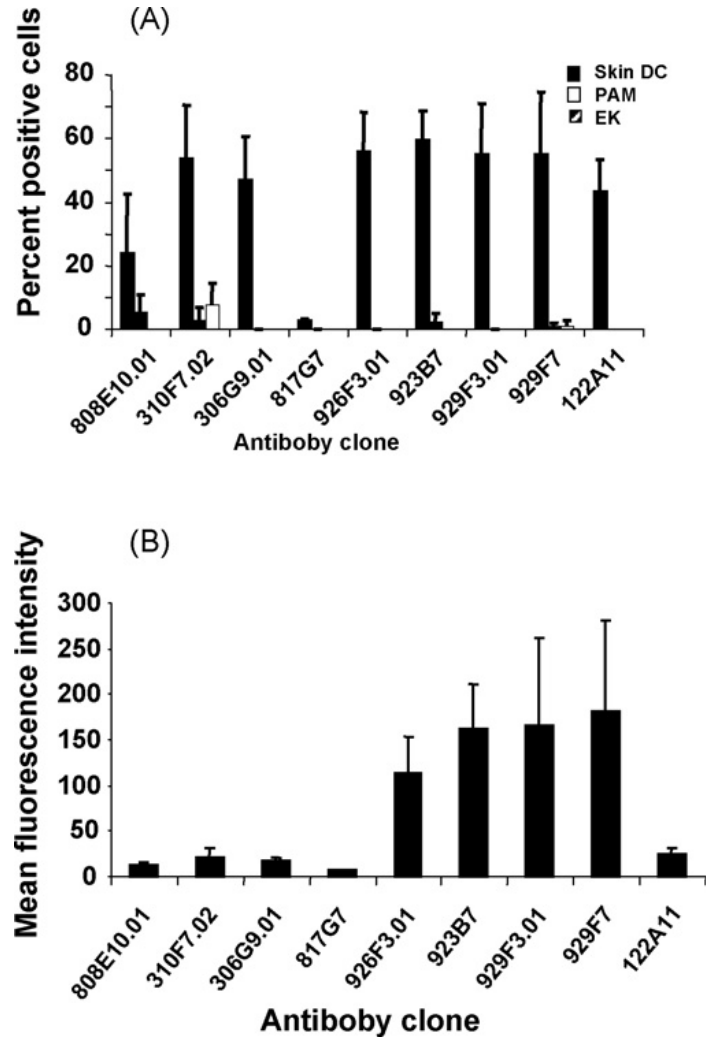

Fig. 4. Multiple clones of anti-human langerin antibodies stain porcine skin DCs but not pulmonary alveolar macrophages (PAMs) or epidermal keratinocytes (EKs). Porcine skin DCs, PAMs and EKs were stained with optimal concentrations of anti-human langerin antibodies and percent positive cells or fluorescence analyzed on a FACS Calibur ${ }^{\mathbb{R}}$ flow cytometer. (A) Percent of cells positive for langerin. (B) Mean fluorescence intensity of langerin-positive skin DCs. Bars represent standard deviation of means. $n=3$ where $n$ represents the number of donor animals used to generate cells and analyzed individually.

sections of porcine skin for immunofluorescent microscopy. Isolated skin DCs readily reacted with 929F3 antibody to human langerin (red, Fig. 7A) and also expressed CD1 (green, Fig. 7B), as further disclosed in Fig. 7C by the yellow color when the images were merged. Similarly, in situ, langerin expressing cells in skin (Fig. 7D) also expressed CD1 (Fig. 7E). Fig. 7F shows the double positive cells (yellow color resulting from the merge of images from Fig. 7D and E) and reveals LCs in situ with cytoplasmic projections (dendrites) characteristic of DCs. Cell nuclei were stained with TOPRO-iodide to outline the

Table 3

Percentage of skin-derived dendritic cells that are Langerhans cells

\begin{tabular}{lc}
\hline Clone & $\%$ LCs $^{\mathrm{a}}$ \\
\hline 808E10.01 & $32.5 \pm 26.3$ \\
310F7.02 & $75.6 \pm 16.0$ \\
306G9.01 & $66.9 \pm 11.8$ \\
817G7 & $3.8 \pm 0.7$ \\
929F3.01 & $74.7 \pm 13.6$ \\
923B7 & $79.7 \pm 9.2$ \\
926G4 & $78.0 \pm 14.1$ \\
929F7 & $77.3 \pm 19.4$ \\
\hline
\end{tabular}

a Langerin positive cells expressed as a percentage of CD1+ CD172+ double positive. skin section and indicates that the langerin/CD1 coexpressing cells were found mainly in the epidermis (Fig. 7G). Anti-human langerin clone 923B7 also stained LCs in skin sections by IF (data not shown).

\subsection{Anti-langerin antibody is internalized into porcine skin $D C$}

Anti-langerin clone 122A11 binds an extracellular epitope of langerin (Fig. 3E) and can be endocytozed into BGs. An analysis of porcine skin DC interaction with this antibody revealed that anti-langerin antibody is rapidly endocytosed, with mean surface expression declining to approximately 50 and $30 \%$ after 15 and $30 \mathrm{~min}$ at $37{ }^{\circ} \mathrm{C}$ respectively when compared to cells incubated on ice for the same length of time (Fig. 8).

\section{Discussion}

We have previously isolated and characterized skin DCs of swine (Bautista et al., 2005, 2002). These cells are distinguished from other porcine mononuclear cells by coexpressing CD1, CD172 and S100 at high levels and no expression of CD14. Swine DCs derived from bone marrow express CD1, CD14 and CD172 (Carrasco et al., 2001) whereas monocyte-derived DCs from peripheral blood are CD172 positive, CD1 dull and CD14 negative (Bautista et al., 2007; Summerfield et al., 2003). All of these DC subsets are also characterized by high expression of MHC class II, CD80/86 and the ability to take up and process antigen and the induction of primary allogenic responses (Bautista et al., 2002, 2007; Carrasco et al., 2001; Summerfield et al., 2003). However, a distinguishing feature of LCs is the expression of langerin (CD207) concentrated in racket-shaped Birbeck granules within the cytoplasm (Valladeau et al., 2000).

Here, we characterize the porcine langerin gene, presenting the full length sequence and comparison with the CD207 sequence from other species presently in the database. Interestingly, where the porcine gene is most closely related to the equine, these two genes are more closely related to the simian species than are the rodent genes to simian. The predicted porcine CD207 protein shares (75\%/86\%) amino acid identity/similarity with human and lesser amino acid identity/similarity to mouse (63\%/77\%) and rat (63\%/76\%) CD207 proteins suggesting that antibodies to human langerin would be more likely to cross react with the porcine protein than antibodies specific for the murine CD207. In fact, binding data for the human protein using some of the anti-human CD207 antibodies tested in swine, predict specificity for the neck region of the protein, just external to the transmembrane region. This region is highly conserved in the simian, equine and porcine genes.

Before testing for protein, we confirmed the presence of langerin mRNA in skin DCs by PCR. These DCs had 105.3fold more langerin-specific mRNA than MoDCs. However, there were detectable levels of langerin mRNA in both EKs and PAMs. EKs contained about $8.5 \%$ residual skin DCs as determined by flow cytometry. Given that PCR amplifies small quantities of RNA, the langerin detected in the EKs 

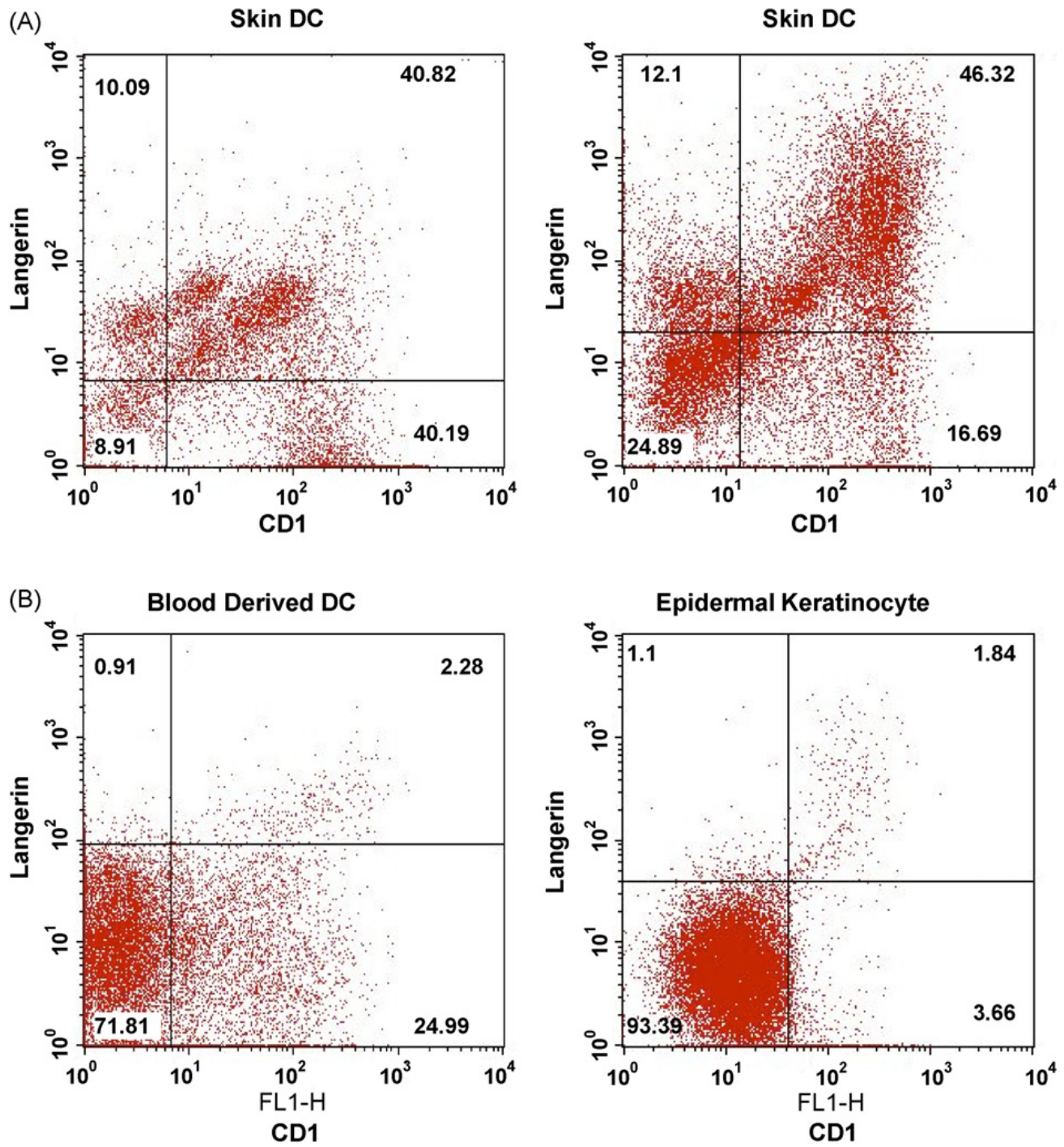

Fig. 5. Double staining of porcine skin-derived DCs, blood DCs and epidermal keratinocytes (EKs) for langerin and CD1. (A) Skin DCs show a significant proportion of skin DCs staining double positive for CD1 and langerin clones 306G9.01 and 929F3 (left and right panel, respectively). (B) EKs from the same biopsy and PBMCs enriched for blood DCs by negative selection, were first stained for CD1, then permeabilized and stained for langerin (clone 929F3). Numbers in each quadrant indicate the percentages of cells. Similar results were obtained from two animals and either anti-langerin antibody clones $929 \mathrm{~F} 3$ or 306G9.01.

could be from the residual skin DCs. Similarly, PAMs had detectable levels of langerin mRNA. LCs are present in the lining of the respiratory tract and could be present in small numbers within the broncho-alveolar lavage cells along with the PAMs. In fact, Valladeau et al. (1999) detected significant levels of langerin in the lungs which they attribute to LCs lining the pulmonary epithelium.

Though LCs have been described in the pig based on the microscopic detection of BGs (Monteiro-Riviere, 1992; Romano and Balaguer, 1991), we could not confirm the presence of these granules in the porcine skin DCs described above (Bautista et al., 2002). Similarly, other researchers have failed to identify these granules in porcine epidermal DCs (Gregg et al., 1995a). This discrepancy could be addressed by identifying antibodies that react with porcine langerin. This C-type lectin is the principal protein component of the BGs and is found exclusively in LCs among skin DCs (Valladeau et al., 2000). Therefore, antibodies against langerin have been used to identify human LCs (Valladeau et al., 2000) and the present study shows porcine skin DC also express CD207/ langerin.

We tested multiple anti-human langerin monoclonal antibodies for cross-reactivity with swine CD207/langerin and identified monoclonal antibodies that reacted with a significant proportion of porcine skin DCs. These antibodies showed very minimal reactivity to blood DCs, PAMs and EKs obtained from the same animals, suggesting that the reactivity was specific for porcine LCs. Based on the coexpression of langerin and CD1, we estimate that LCs make up at least $70 \%$ of DCs isolated from porcine skin. LCs are known to be the principal DCs of the skin and the langerin-negative skin DCs are probably of dermal origin, which are known to share other characteristics with LCs (Lenz et al., 1993).

We subsequently used these crossreactive antihuCD207/langerin antibodies to identify LCs in situ in porcine skin sections by IHC and IF. Cells expressing both CD207 and CD1, with the characteristic dendrites of DCs, were found predominantly in the epidermis. This is in 
(A)

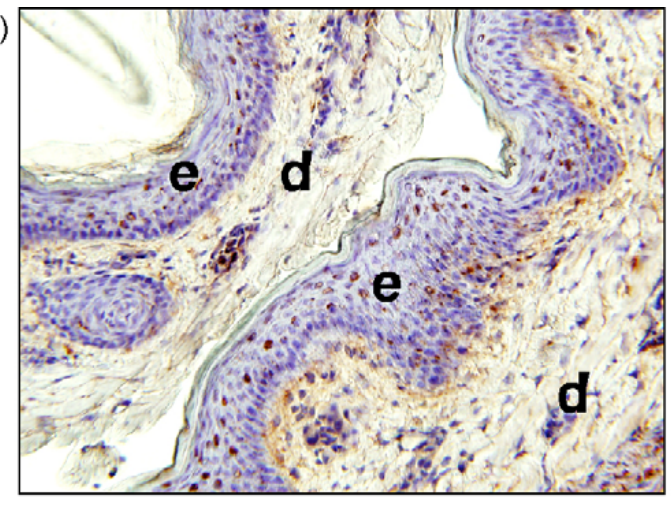

(C)

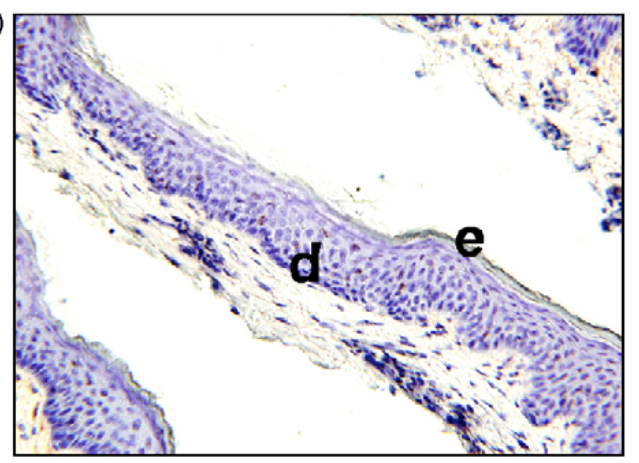

(B)

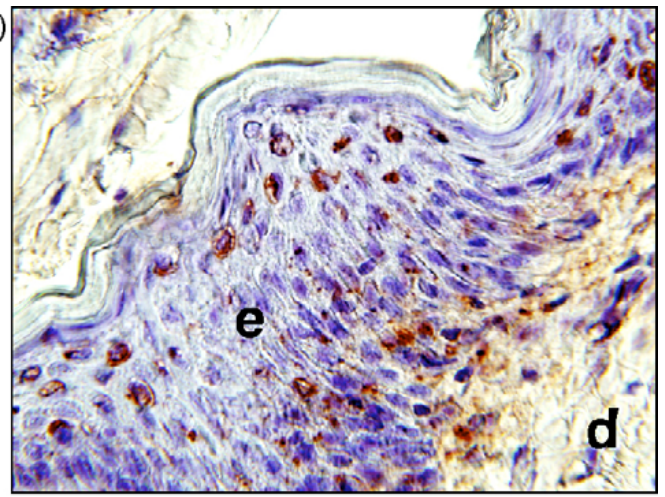

(D)

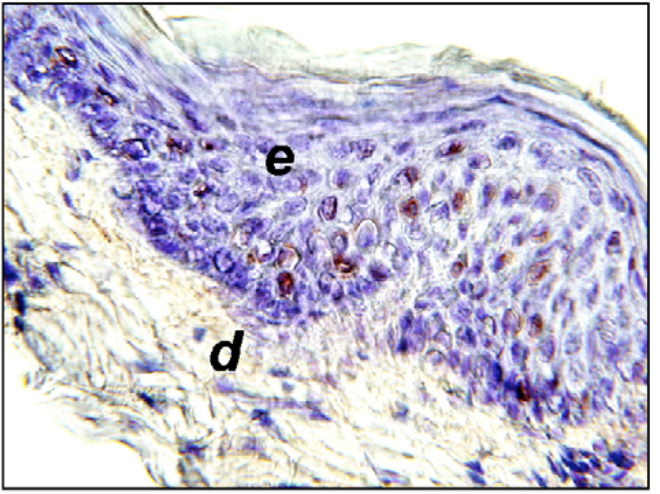

(E)

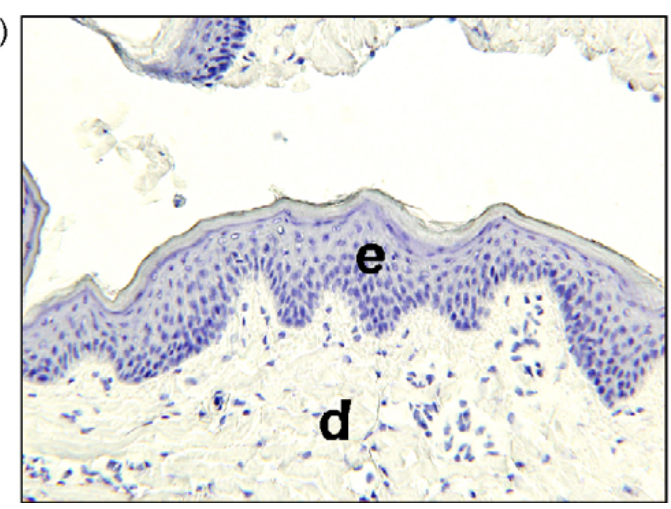

Fig. 6. Immunohistochemical staining of porcine LCs in situ in skin cryosections: Cryosections of porcine skin were fixed and permeabilized before staining with optimal concentrations of specific antibodies or isotype control. Positive cells stained reddish brown, are located mainly in the epidermis. (A and B)

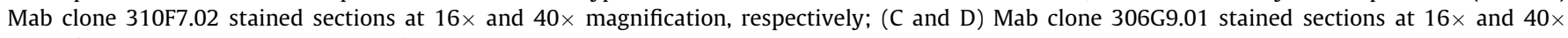
magnification, respectively. (E) Lack of staining with isotype control Mab. $\mathrm{e}=$ epidermis; $\mathrm{d}=$ dermis.

agreement with the electron microscopic description of LCs in pig skin by Romano and Balaguer (1991). These investigators identified cells with characteristic BGs in the basal and suprabasal layers of the porcine epidermis. This is also consistent with data from human and other species in which LCs are typically found in the epidermis and stratified epithelia but can migrate to regional lymph nodes upon activation (Lukas et al., 1996).

Our previous report failed to identify BGs by electron microscopy, implying the absence of LCs (Bautista et al., 2002). Various factors could account for this including the failure to visualize BGs in certain studies due to the age of the donor animals or the handling or processing of samples for electron microscopy. Indeed, LCs are rare in the skin of young pigs (Monteiro-Riviere, 1992) and LCs may lose the characteristic BGs in the maturation process in vitro
(Romani et al., 1989). A more definitive way to identify LCs in porcine is by the reactivity of these antibodies against langerin. In fact, analysis of langerin expression by DC of the skin by immunohistochemistry presented in this report (Fig. 6) shows very similar staining as we previously reported for CD1, CD172 and Class II MHC in skin sections when considering either the epidermal location of cells expressing these proteins or the number (Bautista et al., 2002). Further, detection of both CD1 and langerin in frozen skin sections shows all cells reacting with either antibody, react with both (Fig. 7). Taken together, these data strongly indicate the vast majority of dendritic cells (CD1/CD172 expressing cells) in the epidermis are expressing langerin and are bonafide Langerhans cells.

Pigs have been proposed as important models in human medicine and potential organ donors for xeno- 
(A)

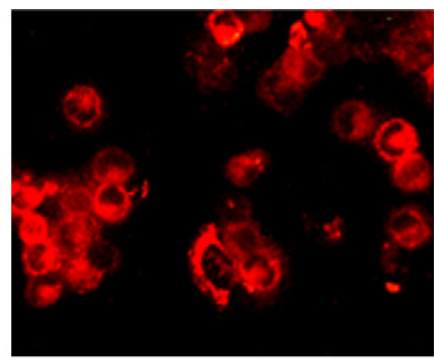

(B)

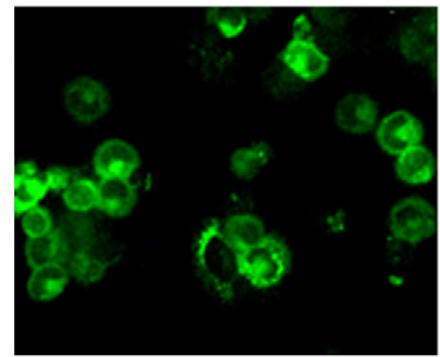

(C)

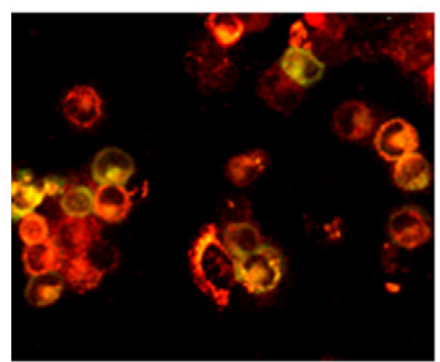

(D)

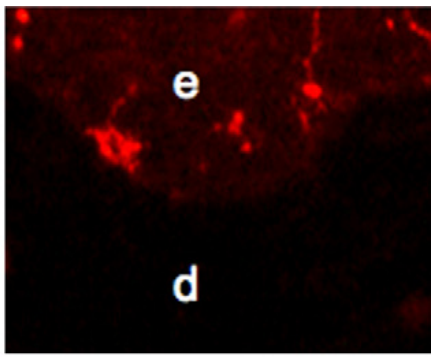

(E)

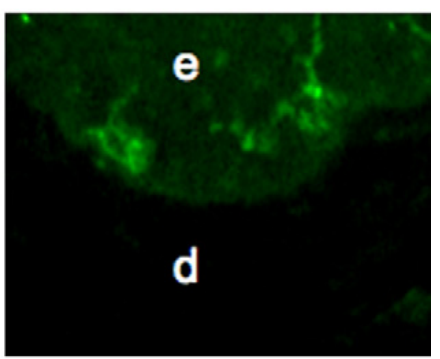

(F)

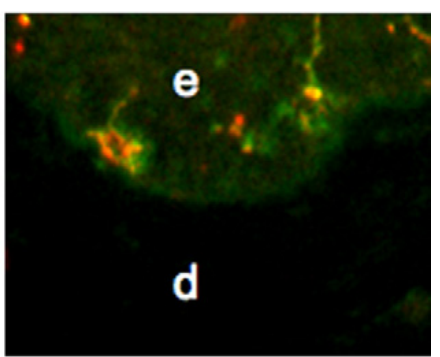

(G)

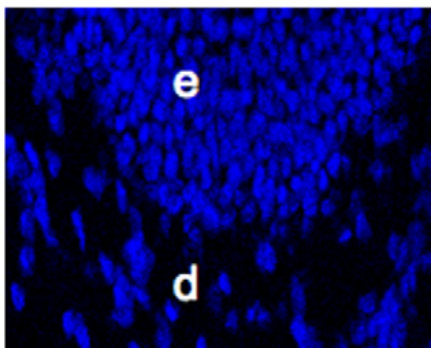

Fig. 7. Immunofluorescent staining of porcine LCs in cytospins of isolated skin DCs and in situ in skin cryosections: Cytospins of skin DCs and cryosections of porcine skin were fixed before staining with optimal concentrations of specific antibodies or isotype control for langerin (clone 929F3) and CD1. Images were captured from same sections for langerin+ cells (red) and CD1+ cells (green) and the images fused to reveal doubly stained cells (yellow). Langerin+ cells (A for cytospins and D for in situ) were also CD1+ (B for cytospins and E for in situ). C and F show a composition of the respective preceding figures. G shows TOPRO-stained nuclei of all cells to reveal the architecture of the skin. $\mathrm{d}=$ dermis; $\mathrm{e}=$ epidermis. Similar results were obtained from two animals. (For interpretation of the references to color in this figure legend, the reader is referred to the web version of the article.)

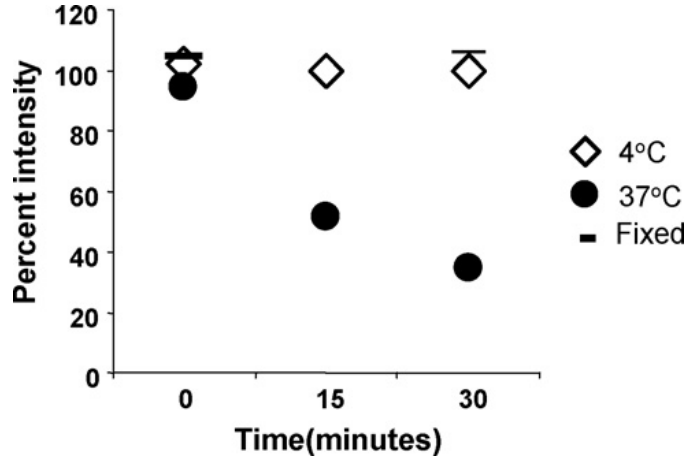

Fig. 8. Anti-langerin antibody clone $122 \mathrm{~A} 11$ is internalized into porcine skin DC at $37^{\circ} \mathrm{C}$. Skin DCs were stained with anti-langerin clone 122A11, followed by biotin anti-mouse IgG, incubated at $37^{\circ} \mathrm{C}$ for indicated time periods and antibody binding revealed with streptavidin-PE. Surface antibody intensity at $37^{\circ} \mathrm{C}(\bullet)$ was expressed as a percentage of that on cells incubated on ice $(\diamond)$ for an equal time. Fixed cells $(-)$ incubated at $37{ }^{\circ} \mathrm{C}$ served as controls for the off-rate the antibody. transplantation because of physiological and immunological similarities with humans (Dunning et al., 1994; Sablinski et al., 1997; Sim et al., 1999). A better understanding of porcine immune responses compared to humans is required in order to fully exploit the potential of pigs for modeling human disease. Knowledge of the role of LCs in porcine immunity is critical to achieving this understanding. Grabbe et al. (1994) showed that inbred miniature pigs possess skin APCs which function in the same way as human and murine LCs. In addition, other studies have shown that porcine skin DCs are important in delayed hypersensitivity reactions (Grabbe et al., 1994; McFarlin and Balfour, 1973) and are involved in allergic contact dermatitis (Vana and Meingassner, 2000). Our data show that porcine skin DCs transcribe CD207/langerin mRNA and express langerin protein, which is reactive with antibodies raised against human langerin and that the majority of DCs in porcine skin epithelium are LCs. 


\section{Acknowledgements}

This work was supported by CRIS \#1940-32000-04800D from the Agricultural Research Service, USDA (WTG) and CRIS \#1123512551 from the Agricultural Research Service, USDA (HD). C. Nfon was the recipient of a Plum Island Animal Disease Center Research Participation Program fellowship, administered by the Oak Ridge Institute for Science and Education (ORISE) through an interagency agreement between the U.S. Department of Energy (DOE) and the U.S. Department of Agriculture (USDA). The authors thank Dr. Celine Chen, Ms. Vandana Vangimalla and Mr. Sebastian Botero (DGIL, BHNRC, BARC) for helpful assistance with sequencing porcine CD207. We would also like to thank Dendritics (Lyon, France) for providing a panel of monoclonal antibodies for testing and Dr. Giorgio Trinchieri, NCI, NIH, Frederick, MD for his advice and consultation. We also thank the animal care staff at the Plum Island Animal Disease Center for their professional support and assistance.

\section{References}

Banchereau, J., Steinman, R.M., 1998. Dendritic cells and the control of immunity. Nature 392, 245-252.

Bautista, E.M., Ferman, G.S., Gregg, D., Brum, M.C., Grubman, M.J., Golde, W.T., 2005. Constitutive expression of alpha interferon by skin dendritic cells confers resistance to infection by foot-and-mouth disease virus. J. Virol. 79, 4838-4847.

Bautista, E.M., Gregg, D., Golde, W.T., 2002. Characterization and functional analysis of skin-derived dendritic cells from swine without a requirement for in vitro propagation. Vet. Immunol. Immunopathol. $88,131-148$.

Bautista, E.M., Nfon, C., Ferman, G.S., Golde, W.T., 2007. IL-13 replaces IL-4 in development of monocyte derived dendritic cells (MoDC) of swine. Vet. Immunol. Immunopathol. 115, 56-67.

Carrasco, C.P., Rigden, R.C., Schaffner, R., Gerber, H., Neuhaus, V., Inumaru, S., Takamatsu, H., Bertoni, G., McCullough, K.C., Summerfield, A., 2001. Porcine dendritic cells generated in vitro: morphological, phenotypic and functional properties. Immunology 104, 175-184.

Douillard, P., Stoitzner, P., Tripp, C.H., Clair-Moninot, V., Ait-Yahia, S., McLellan, A.D., Eggert, A., Romani, N., Saeland, S., 2005. Mouse lymphoid tissue contains distinct subsets of langerin/CD207 dendritic cells, only one of which represents epidermal-derived Langerhans cells. J. Invest. Dermatol. 125, 983-994.

Drummond, A.J., Rambaut, A., 2007. BEAST: Bayesian evolutionary analysis by sampling trees. BMC Evol. Biol. 7, 214.

Dunning, J.J., White, D.J., Wallwork, J., 1994. The rationale for xenotransplantation as a solution to the donor organ shortage. Pathol. Biol. (Paris) 42, 231-235.

Grabbe, S., Fishbein, J.M., Sachs, D.H., Flotte, T.J., Granstein, R.D., 1994. Characterization of cutaneous antigen presentation in partially inbred miniature swine. Exp. Dermatol. 3, 276-282.

Gray, G.M., Yardley, H.J., 1975. Different populations of pig epidermal cells: isolation and lipid composition. J. Lipid Res. 16, 441-447.

Gregg, D.A., Mebus, C.A., Schlafer, D.H., 1995a. Early infection of interdigitating dendritic cells in the pig lymph node with African swine fever viruses of high and low virulence: immunohistochemical and ultrastructural studies. J. Vet. Diagn. Invest. 7, 23-30.

Gregg, D.A., Schlafer, D.H., Mebus, C.A., 1995b. African swine fever virus infection of skin-derived dendritic cells in vitro causes interference with subsequent foot-and-mouth disease virus infection. J. Vet. Diagn. Invest. 7, 44-51.

Hollis, D.E., Lyne, A.G., 1972. Acetylcholinesterase-positive langerhans cells in the epidermis and wool follicles of the sheep. J. Invest. Dermatol. 58, 211-217.
Khalil, H.M., Nitiuthai, S., Allen, J.R., 1982. Alkaline phosphatase-positive Langerhans cells in the epidermis of cattle. J. Invest. Dermatol. 79, 47-51.

Lenz, A., Heine, M., Schuler, G., Romani, N., 1993. Human and murine dermis contain dendritic cells. Isolation by means of a novel method and phenotypical and functional characterization. J. Clin. Invest. 92, 2587-2596.

Lukas, M., Stossel, H., Hefel, L., Imamura, S., Fritsch, P., Sepp, N.T., Schuler, G., Romani, N., 1996. Human cutaneous dendritic cells migrate through dermal lymphatic vessels in a skin organ culture model. J. Invest. Dermatol. 106, 1293-1299.

Marchal, T., Saint-Andre, I., Magnol, J.P., Dezutter-Dambuyant, C., Schmitt, D., 1995. Dendritic cells in dogs and cats: models of study in human pathology. Pathol. Biol. (Paris) 43, 910-920.

McFarlin, D.E., Balfour, B., 1973. Contact sensitivity in the pig. Adv. Exp. Med. Biol. 29, 539-544.

Monteiro-Riviere, N.A., 1992. Langerhans cell granules in porcine epidermis. J. Anat. 180 (Pt 3), 555-556.

Paillot, R., Laval, F., Audonnet, J.C., Andreoni, C., Juillard, V., 2001. Functional and phenotypic characterization of distinct porcine dendritic cells derived from peripheral blood monocytes. Immunology 102, 396-404.

Perez-Torres, A., Ustarroz-Cano, M., 2001. Demonstration of birbeck (Langerhans cells) granules in the normal chicken epidermis. J. Anat. 199, 493-497.

Romani, N., Ebner, S., Tripp, C.H., Flacher, V., Koch, F., Stoitzner, P., 2006. Epidermal Langerhans cells-changing views on their function in vivo. Immunol. Lett. 106, 119-125.

Romani, N., Lenz, A., Glassel, H., Stossel, H., Stanzl, U., Majdic, O., Fritsch, P., Schuler, G., 1989. Cultured human Langerhans cells resemble lymphoid dendritic cells in phenotype and function. J. Invest. Dermatol. 93, 600-609.

Romano, J., Balaguer, L., 1991. Ultrastructural identification of Langerhans cells in normal swine epidermis. J. Anat. 179, 43-46.

Sablinski, T., Gianello, P.R., Bailin, M., Bergen, K.S., Emery, D.W., Fishman, J.A., Foley, A., Hatch, T., Hawley, R.J., Kozlowski, T., Lorf, T., Meehan, S., Monroy, R., Powelson, J.A., Colvin, R.B., Cosimi, A.B., Sachs, D.H., 1997. Pig to monkey bone marrow and kidney xenotransplantation. Surgery 121, 381-391.

Sim, K.H., Marinov, A., Levy, G.A., 1999. Xenotransplantation: a potential solution to the critical organ donor shortage. Can. J. Gastroenterol. 13, 311-318.

Stambach, N.S., Taylor, M.E., 2003. Characterization of carbohydrate recognition by langerin, a C-type lectin of Langerhans cells. Glycobiology 13, 401-410.

Summerfield, A., Guzylack-Piriou, L., Schaub, A., Carrasco, C.P., Tache, V., Charley, B., McCullough, K.C., 2003. Porcine peripheral blood dendritic cells and natural interferon-producing cells. Immunology 110, 440449.

Sung, S.S., Fu, S.M., Rose Jr., C.E., Gaskin, F., Ju, S.T., Beaty, S.R., 2006. A major lung CD103 (alphaE)-beta7 integrin-positive epithelial dendritic cell population expressing Langerin and tight junction proteins. J. Immunol. 176, 2161-2172.

Valladeau, J., Duvert-Frances, V., Pin, J.J., Dezutter-Dambuyant, C., Vincent, C., Massacrier, C., Vincent, J., Yoneda, K., Banchereau, J., Caux, C., Davoust, J., Saeland, S., 1999. The monoclonal antibody DCGM4 recognizes Langerin, a protein specific of Langerhans cells, and is rapidly internalized from the cell surface. Eur. J. Immunol. 29, 26952704.

Valladeau, J., Ravel, O., Dezutter-Dambuyant, C., Moore, K., Kleijmeer, M., Liu, Y., Duvert-Frances, V., Vincent, C., Schmitt, D., Davoust, J., Caux, C., Lebecque, S., Saeland, S., 2000. Langerin, a novel C-type lectin specific to Langerhans cells, is an endocytic receptor that induces the formation of Birbeck granules. Immunity 12 , 71-81.

Vana, G., Meingassner, J.G., 2000. Morphologic and immunohistochemical features of experimentally induced allergic contact dermatitis in Gottingen minipigs. Vet. Pathol. 37, 565-580.

Verdijk, P., Dijkman, R., Plasmeijer, E.I., Mulder, A.A., Zoutman, W.H., Mieke Mommaas, A., Tensen, C.P., 2005. A lack of Birbeck granules in Langerhans cells is associated with a naturally occurring point mutation in the human Langerin gene. J. Invest. Dermatol. 124, 714-717. 